\title{
Biochemical characterization of crystallins from pigeon lenses: structural and sequence analysis of pigeon $\delta$-crystallin ${ }^{1}$
}

\author{
Shyh-Horng Chiou ${ }^{\mathrm{a}, \mathrm{b}}$, Chin-Chun Hung ${ }^{\mathrm{b}}$ and Chou-Wen Lin ${ }^{\mathrm{a}}$ \\ ${ }^{a}$ Laboratory of Crystallin Research, Institute of Biochemical Sciences, National Taiwan University, Taipei (Taiwan) \\ and ${ }^{b}$ Institute of Biological Chemistry, Academia Sinica, Taipei (Taiwan)
}

(Received 7 July 1992)

(Revised version received 18 September 1992)

Key words: Pigeon lens; $\delta$-Crystallin; Argininosuccinate lyase; Glycogen; Polymerase chain reaction; Sequence comparison; (C. livia)

Crystallins from pigeon eye lenses were isolated and purified by gel-permeation chromatography and characterized by gel electrophoresis, amino-acid composition and sequence analysis. $\alpha$ - and $\beta$-crystallins could be obtained in relatively pure forms by single-step size-exclusion chromatography whereas an extra step of ion-exchange chromatography was needed for the separation of $\delta$-crystallin from the $\beta$-crystallin fraction. In contrast to most characterized vertebrate species, a large amount of glycogen is eluted as a high molecular form in the first peak of the gel filtration column. Pigeon $\delta$-crystallin, similar to duck and reptilian $\delta$-crystallins, exists as a tetrameric structure of about $200 \mathrm{kDa}$ in the native form and is composed of one major subunit of $50 \mathrm{kDa}$ with heterogeneous isoelectric points spreading in a range of 4.7 to 6.8. In contrast to those obtained from duck, goose and caiman, $\delta$-crystallin isolated from the pigeon lens possessed very little argininosuccinate lyase activity. However, pigeon $\delta$-crystallin can still cross-react with the antibody against enzymically active duck $\delta$-crystallin as revealed by the sensitive immunoblotting technique. It was also shown that the $\delta$-crystallin content of the total pigeon soluble proteins decreased with the age of the animal. Structural analysis of purified $\delta$-crystallin fraction was made with respect to its amino-acid composition and protein primary sequence. $\mathrm{N}$-terminal sequence analysis indicated the presence of blocked amino-termini in all crystallin fractions of pigeon lenses. Therefore, a sequence analysis of PCR (polymerase chain reaction) amplified $\delta$-crystallin cDNA was employed to deduce the protein sequence of this crystallin. Structural comparison of $\delta$-crystallin sequences from pigeon, chicken and duck lenses casts some doubts on the recent claim that His-89 $\rightarrow$ Gln mutation in the chicken $\delta$-crystallin may account for the loss of argininosuccinate lyase activity in this avian species, as compared to high enzymic activity in the duck crystallin (Barbosa et al. (1991) J. Biol. Chem. 266, 5286-5290).

\section{Introduction}

The lens crystallins of vertebrates comprise a complex group of conserved structural proteins with distant evolutionary relationships $[1,2]$. Previous characterization and classification of lens crystallins were based on charge and size properties of crystallins with some biochemical methods and emphasis was also directed to mammalian lenses due to their large size and easy availability [3]. The difficulties arising from the characterization of avian crystallins lie in the relatively soft consistency and great plasticity associated with small

Correspondence to: S.-H. Chiou, Institute of Biological Chemistry, Academia Sinica, P.O. Box 23-106, Taipei, Taiwan.

1 The sequence data of the pigeon $\delta$-crystallin gene have been deposited in the EMBL Data Library under the accession number X66404. and soft avian lenses. However, the recent sequence analysis and comparison of various crystallin polypeptides have revealed salient features in structural similarities between crystallins and some metabolic enzymes [4] from the avian class. Therefore, it is of interest to study and compare crystallins of the available species in the Aves to shed some light on the mechanism underlying the process of crystallin diversification and the possible enzymic role of these presumably structural proteins inside avian lenses.

Recently, we have studied avian $\epsilon$ - and $\delta$-crystallins with genuine enzymic activity from duck and ostrich lenses, respectively, establishing a close inter-relatedness between these presumably structural proteins and those authentic enzymes isolated from other tissues in regard to their kinetic mechanisms [5-7]. In the course of a systematic screening on the presence of these enzymic crystallins in avian species, we have found some interesting aspects regarding the lens compo- 
nents present in the pigeon as compared to other poultry birds. In this report, we have furnished biochemical informations on the lens composition isolated from the pigeon, which has not been studied biochemically in details to date. Some structural information obtained by the application of a facile PCR technique to the determination of pigeon $\delta$-crystallin sequence is also provided to reveal some insights on the structural residues important for the enzymic activity associated with this unique crystallin in some avian species of the Anatidae family, such as duck and goose [8].

\section{Materials and Methods}

\section{Isolation of lens crystallins}

The lenses of common domestic pigeons (Columba livia) of about 1-month and 1-year-old were obtained from local livestock markets (Taipei, Taiwan). Other sources of lenses, including those of chicken, goose, shark, caiman and duck, were easily obtained from local markets in the Taipei area, except ostrich lenses from South Africa.

The preparation and fractionation of lens homogenates from avian species were essentially according to the previous reports $[5,6]$. Gel permeation and anion-exchange chromatography of total crude crystallins were carried out on Fractogel TSK HW-55(S) and DEAE-650(M) columns, respectively. Crude crystallins dissolved in the starting buffer of $0.05 \mathrm{M}$ ammonium bicarbonate with $0.5 \mathrm{mM}$ EDTA ( $\mathrm{pH} 7.7$ ) were applied to the column equilibrated in the same buffer. Ion-exchange chromatography was carried out in two steps: (A), elution with starting buffer and (B), elution with a $500 \mathrm{ml}$ linear gradient of $0.05-0.5 \mathrm{M}$ ammonium bicarbonate $(\mathrm{pH}$ 7.7). Native molecular masses of the eluted fractions were determined on the same TSK HW-55 column $(2.5 \times 115 \mathrm{~cm})$ using the following standard proteins: thyroglobulin $(670 \mathrm{kDa})$, catalase $(240$ $\mathrm{kDa})$, transferrin $(80 \mathrm{kDa})$, ovalbumin $(45 \mathrm{kDa})$ and soybean trypsin inhibitor $(20 \mathrm{kDa})$. The separated subfractions were rechromatographed once before they were taken for amino-acid analyses.

\section{Gel electrophoresis and immunoblotting of pigeon crys-} tallins

SDS-PAGE (5\% stacking/14\% resolving gel) was performed as described with some modifications [9]. Isoelectric focusing in 5\% polyacrylamide gel containing $2.8 \%(\mathrm{v} / \mathrm{v})$ carrier ampholytes of $\mathrm{pH} 3.5-10$ was carried out on a slab gel with the incorporation of $0.1 \%$ 2-mercaptoethanol and $6 \mathrm{M}$ urea. The gel was fixed in $12.5 \%$ trichloroacetic acid, washed several times with $20 \%$ methanol $/ 7 \%$ acetic acid and stained with Coomassie blue. For immunoblotting detection of $\delta$-crystallin, the gels were subjected to electroblotting to a nitrocellulose membrane after SDS-PAGE fol- lowed by immunological analysis using rabbit antiserum against duck $\delta$-crystallin (electroeluted $\delta$-crystallin band from SDS-PAGE) and stained with horseradish peroxidase conjugated goat anti-rabbit IgG (Sigma, St. Louis, MO, USA).

\section{Enzyme activity assay}

Argininosuccinate lyase activity of duck, chicken and goose $\delta$-crystallins plus total lens homogenates from various species was assayed at $25^{\circ} \mathrm{C}$. The formation of fumarate in the forward direction and disappearance of fumarate in the reverse direction were monitored continuously at $240 \mathrm{~nm}$ in a Hitachi UV-Visible (Model U-2000) spectrophotometer. In a standard assay for the forward reaction, the reaction mixture contained 50 $\mathrm{mM}$ Tris- $\mathrm{HCl}$ buffer ( $\mathrm{pH} 7.5), 0.2 \mathrm{mM}$ argininosuccinate and an appropriate amount of $\delta$-crystallin in a total volume of $1 \mathrm{ml}$. 1 unit of enzyme activity was defined as an initial rate of $1 \mu \mathrm{mol}$ of fumarate released per min under the assay conditions. A molar absorption coefficient of $2.44 \cdot 10^{3} \mathrm{M}^{-1} \mathrm{~cm}^{-1}$ for fumarate was used for calculation. Argininosuccinate and authentic argininosuccinate lyase from the bovine liver was obtained from Sigma.

\section{Amino-acid and sequence analysis}

The amino-acid compositions were determined with the Beckman high-performance amino-acid analyzer (model 6300) using a single-column system. The dialyzed and lyophilized protein samples were hydrolyzed at $160^{\circ} \mathrm{C}$ on a dry heating block using Teflon-Pyrex re-usable hydrolysis tubes (The Continuity Enterprise, Taipei, Taiwan) with constant-boiling $6 \mathrm{M} \mathrm{HCl}$ for 45 min. The special rapid procedure for the preparation of protein hydrolysates using microwave irradiation before amino-acid analysis was also performed essentially according to the previous report [10].

$\mathrm{N}$-terminal sequence analysis was carried out by automated Edman degradation with a pulsed-liquid phase sequenator (Model 477A, Applied Biosystems, Foster City, CA, USA). The lyophilized crystallin samples each containing about $1-5 \mathrm{nmol}$ of protein were dissolved in $200 \mu \mathrm{l}$ of $0.1 \%$ trifluoroacetic acid (TFA) or $0.1 \% \mathrm{SDS} / 0.1 \%$ TFA $(1: 1 \mathrm{v} / \mathrm{v})$ and $10 \mu \mathrm{l}$ each for sequence determinations.

\section{Carbohydrate determination}

The amount of glycogen content was determined by measuring the glucose release after acid hydrolysis of total extract and glycogen fraction from gel filtration with sulfuric acid [11]. The results were expressed as \% content $(w / w)$ of the soluble lens homogenate.

\section{Protein concentration determinations}

The protein concentrations of crystallin solutions were first analyzed on the basis of protein content 


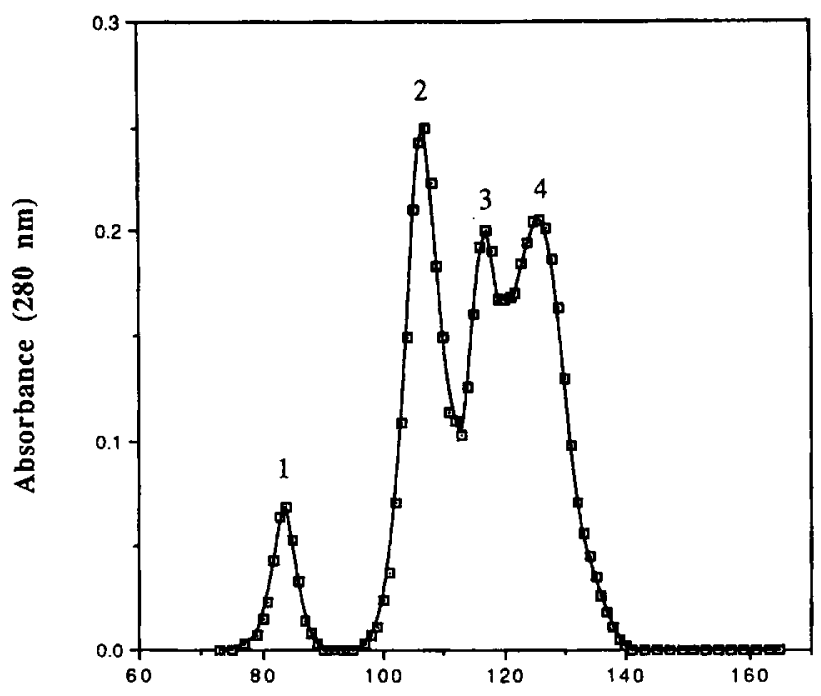

Fraction Number

Fig. 1. Isolation and fractionation of pigeon lens extract. Gel-permeation chromatography on Fractogel TSK HW- $55(\mathrm{~S})(3 \times 95 \mathrm{~cm}$ column) of lens extract (1-month-old lenses) was carried out as described before [6]. The column eluates $(3.8 \mathrm{ml} /$ tube per $4.7 \mathrm{~min})$ were monitored for absorbance at $280 \mathrm{~nm}$. Four major fractions ( $\mathrm{Pg}-1$ to $\mathrm{Pg}-4$ as indicated on the top of each peak fraction) were obtained. The estimation of native molecular masses for each fraction was as described in Materials and Methods. The four peak fractions were checked for the subunit compositions by SDS-PAGE, as shown in Fig. 2A. determined by amino-acid analysis. The absorption coefficient $(1 \mathrm{mg} / \mathrm{ml}$, at $280 \mathrm{~nm})$ was then determined for each purified crystallin. The determined values for the $\alpha-, \beta$ - and $\delta$-crystallins of pigeon lenses were similar to those for bovine and ostrich crystallins reported previously $[6,12]$. The absorption coefficients for each crystallin fraction were employed in the estimation of protein concentrations.

Construction of $c D N A$ and sequence analysis of $\delta$-crystallin by PCR

To obtain a full-length $\delta$-crystallin genes of pigeon lens, lens poly(A) ${ }^{+}$RNA of $C$. livia was purified using the QuickPrep mRNA preparation kit (Pharmacia, Uppsala, Sweden) and then used to the synthesis of cDNA library by RiboClone cDNA synthesis system (Promega, Madison, WI, USA). Two oligonucleotide primers with opposing orientations (covering $5^{\prime}$ - and $3^{\prime}$-coding regions of duck $\delta 1$ and $\delta 2$-crystallin clones [13]), the forward, 5'-ACG TC(C/G) TCT AGA GAT GGC ATC-3' and the reverse 5'-GGG AAA GCT TCC CCA CAC TCT AAGC-3', was first synthesized, each containing $X b a I$ and HindIII sites, respectively.

PCR reactions were carried out in a $100 \mu$ l volume containing $100 \mathrm{ng}$ of template, $500 \mathrm{ng}$ of each primer, $0.225 \mathrm{mM}$ of each dNTP, 2.5 units of Taq polymerase and other buffer components as recommended by
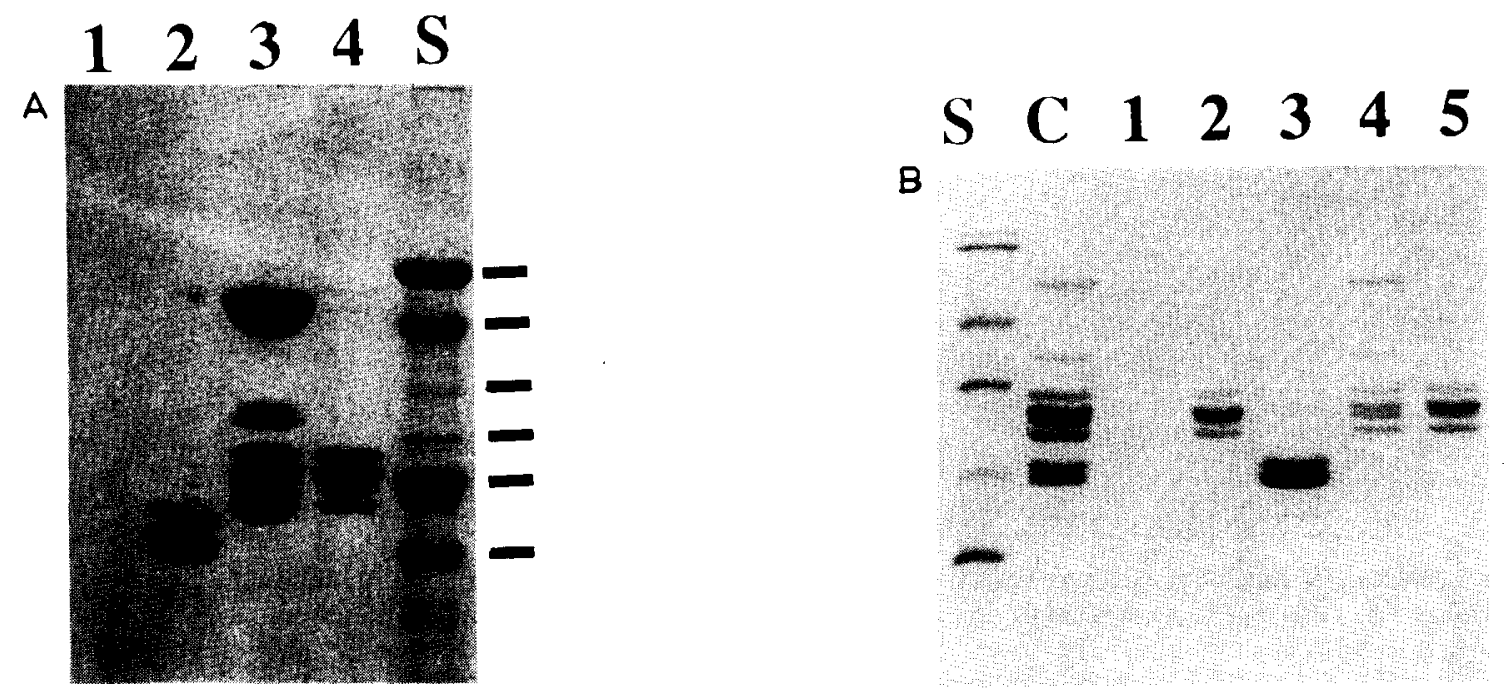

Fig. 2. Gel electrophoresis of fractionated pigeon crystallins under denaturing conditions (SDS-PAGE) in the presence of $5 \mathrm{mM}$ dithiothreitol. (A), 1-month-old pigeon lens fractions. Lanes $\mathbf{S}$, standard proteins used as molecular mass markers with positions indicated by bars on the right (in $\mathrm{kDa}$ ): bovine serum albumin (66), ovalbumin (45), lactate dehydrogenase (37), carbonic anhydrase (30), trypsinogen (25) and soybean trypsin inhibitor (20). Lanes 1-4 are four peak fractions (Fig. 1) corresponding to Pg-1 to Pg-4. The gel was stained with Coomassie blue. Note that Pg-1 of lane 1 is carbohydrate in nature and cannot be stained. Lane $3(\mathrm{Pg}-3)$ is a mixture of $\delta$ - and $\beta$-crystallins. $\delta$-Crystallin (50 kDa band in lane 3 ) was estimated to constitute about $31 \%$ of total lens proteins by densitometry of the Coomassie-blue-stained protein bands. (B), 1 -year-old pigeon lens fractions. Electrophoretic conditions was similar to (A), except that in Lane $\mathrm{S}$ trypsinogen $(25 \mathrm{kDa})$ marker protein was deleted and replaced by lysozyme (14 kDa): Lane C, crude lens extract; lane 1, Pg-1; lanes 2 and 5, Pg-4; lane 3, Pg-2; and lane 4, Pg-3 fraction. $\delta$-Crystallin of this older extract was estimated to be only $4.2 \%$ of total proteins by densitometry of crude extract (lane C). 
Promega. The reactions were subjected to 40 cycles of heat denaturation at $94^{\circ} \mathrm{C}$ for $1.5 \mathrm{~min}$, annealing the primers to the DNA at $50^{\circ} \mathrm{C}$ for $1 \mathrm{~min}$, DNA-chain-extension with $T a q$ polymerase at $72^{\circ} \mathrm{C}$ for $3 \mathrm{~min}$, followed by a final extension at $72^{\circ} \mathrm{C}$ for $10 \mathrm{~min}$. The PCR products were separated on a $1.5 \%$ agarose gel, electroeluted, and digested with $X b a \mathrm{I}$ and HindIII. The DNA fragment was subcloned into pUC19 previously digested with these two enzymes, and then transformed into Escherichia coli JM109. Plasmids purified from the transformed cells were prepared for nucleotide sequencing by the dideoxy chain-termination method [14].

\section{Results and Discussion}

We have characterized the crystallins from several different species encompassing one class of invertebrates [15] and five major classes of vertebrates [16-19] with the aim of searching for their evolutionary relatedness and phylogenetic relationships on the basis of their protein- and gene-structures. The different classes of crystallins not only vary between species but are also differentially expressed during lens development. The bird lens differs at least quantitatively from other species of lenses in several respects [20]. It has a very low proportion of protein. It is neither pigmented nor fluorescent. It is very soft throughout life, never developing the hard nucleus of many mammalian and piscine lenses. It appears that many of these properties may be correlated with the presence in the bird lens of some unique components, such as glycogen [21] and $\delta$-crystallin (previously called FISC, first important soluble crystallin) [22], which constitutes about $30-60 \%$ of most avian lens proteins. Especially noteworthy is the association of $\delta$-crystallin with genuine argininosuccinate lyase ( $\mathrm{L}$-argininosuccinate arginine-lyase, $\mathrm{EC}$ 4.3.2.1) activity $[4,6-8]$ in some of the avian species, one of the key enzymes in the urea cycle. This has prompted us to look for glycogen and this major crystallin in the pigeon lens.

Biochemical characterization of various crystallins eluting from a gel-permeation column

Fig. 1 shows the elution pattern of pigeon lens extract. Four peaks were obtained and identification of each crystallin fraction from the column was made by the subunit analysis using SDS-PAGE, as shown in Fig. $2 \mathrm{~A}$. It is very unexpected to find that the first peak (with an approximate molecular mass of 3000 and 200 $\mathrm{kDa}$ as estimated from protein and dextran markers, respectively) could not be stained with Coomassie blue
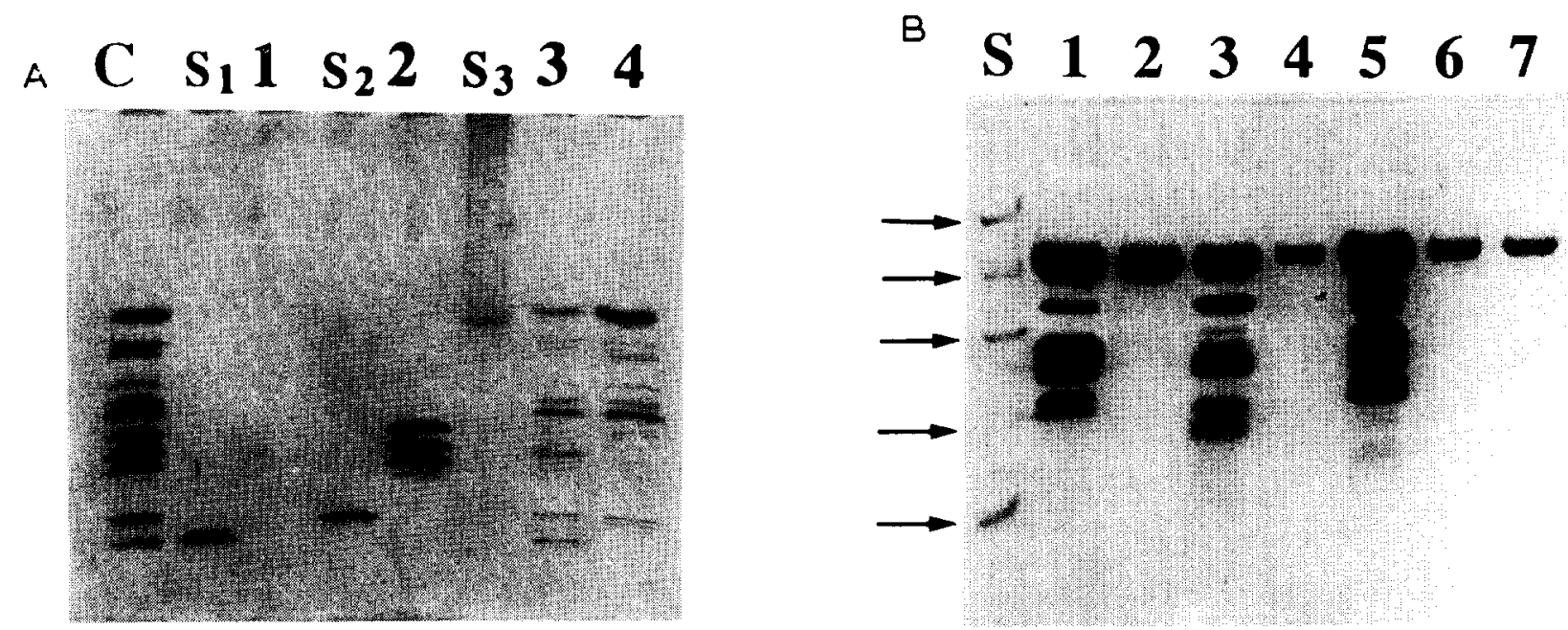

Fig. 3. (A), isoelectric focusing of pigeon lens extract and fractionated crystallins under denaturing conditions. Lanes 1-4 correspond to four peak fractions of Fig. 1 and lane $\mathrm{C}$ denotes the pattern for crude lens extract. The slab gel contained $6 \mathrm{M}$ urea and $0.1 \% 2$-mercaptoethanol and the eleztrophoresis was run at an initial voltage of $200 \mathrm{~V}$ for $6 \mathrm{~h}$ until a final current of less than $0.5 \mathrm{~mA}$ was reached. The approximate isoelectric points of various crystallin polypeptides were estimated from $\mathrm{p} I$-calibration standard proteins of lanes $\mathrm{S}_{1}, \mathrm{~S}_{2}$ and $\mathrm{S}_{3}$ with $\mathrm{p} I 4.6,5.1$ and 6.6, respectively (Sigma). $\mathrm{p} I$ values of 5.3-5.9, 4.7-6.8, 5.1-6.8 were estimated for $\mathrm{Pg}-2$ to $\mathrm{Pg}-4$, respectively. The gel was stained with Coomassie blue. Pg-1 of lane 1 is glycogen and cannot be stained. It is to be noted that the purified pigeon $\delta$-crystallin (Fig. 3B) from anion-exchange chromatography also showed heterogeneity similar to those published for duck and chicken $\delta$-crystallins [7]. (B), gel electrophoresis of purified $\delta$-crystallins isolated from pigeon, chicken and duck lens extracts from anion-exchange chromatography under denaturing conditions (SDS-PAGE) in the presence of $5 \mathrm{mM}$ dithiothreitol. Lanes S, standard proteins used as molecular mass markers with electrophoretic positions indicated by arrows on the left (in $\mathrm{kDa}$ ): bovine serum albumin (66), ovalbumin (45), carbonic anhydrase (30), soybean trypsin inhibitor (20) and lysozyme (14). Lanes 1 and 2, pigeon crude lens extract and purified $\delta$-crystallin, respectively; lanes 3 and 4 , chicken crude lens extract and purified $\delta$-crystallin, respectively; lanes $5-6$, duck crude lens extract and purified $\delta$-crystallin, respectively; lane 7 , purified pigeon $\delta$-crystallin. $\delta$-Crystallins from three species were separated from contaminant $\beta$-crystallins by anion-exchange chromatography. 
(vide infra). The characteristic and well-separated patterns of doublet bands for $\alpha$-crystallin (Fig. 2A, lane 2) are clearly shown in the second peak of about $450 \mathrm{kDa}$. Similar to mammalian $\alpha$-crystallin fraction and contrary to the cases of fish and frog crystallins [16,17], the pigeon $\alpha$-crystallin is well-separated and not contaminated with $\beta$ - and other classes of crystallins. The native molecular masses for the third and fourth peaks corresponding to $\delta / \beta$ and $\beta$-crystallins were estimated to be about 200 and $110 \mathrm{kDa}$ respectively, which are very similar to the corresponding fractions characterized for these crystallins from the swan lens [23], reflective of the conservative nature of molecular size distribution of these crystallin classes within the same bird class.

From SDS-PAGE of Fig. $2 \mathrm{~A}$ it is clearly seen that $\delta$-crystallin has co-eluted with $\beta$-crystallin in peak 3 (lane 3), hence anion-exchange chromatography on a TSK DEAE-650 column, similar to those employed for the purification of $\delta$-crystallins from the duck and ostrich extracts $[5,6]$, was used for the purification of pigeon $\delta$-crystallin (data not shown). A good separation of this major crystallin from other $\beta$-crystallins can be obtained by such a single-step purification. It is also noted that another enzymic $\epsilon$-crystallin with prominent lactate dehydrogenase activity is absent in this flying bird [5,7], in contrast to its presence in some aquatic birds of the Anatidae family [23]. In Fig. 2B, we have shown the crystallin composition of 1-year-old pigeon lens by SDS-PAGE, showing a much less $\delta$-crystallin contribution in this older lens $(4.2 \%(\mathrm{w} / \mathrm{w})$ by densitometry on SDS-PAGE of soluble proteins) as compared to that in the 1-month-old young lens $(31 \%)$. This would indicate that the expression of $\delta$-crystallin in the pigeon is strictly regulated developmentally.

The charge heterogeneity or the multiplicity of crystallin subunits can still be detected in the isoelectricfocusing gel containing $6 \mathrm{M}$ urea (Fig. 3A), in spite of the apparent size homogeneity of $\delta$-crystallins isolated from various avian species (Fig. 3B). $\alpha$-Crystallin (peak 2 of Fig. 1, lane 2 of Fig. 3A) shows one minor and three major bands with low $\mathrm{p} I$ (5.3-5.9) corresponding to well-characterized $\alpha$-A and $\alpha$-B chains $[3,18]$. The isoelectric-focusing patterns of $\delta$ - and $\beta$-crystallins were very complex and the $\mathrm{p} I$ values were estimated to be in the range of 4.7-6.8. These multiple bands have also been reported for ostrich and caiman $\delta$-crystallins $[6,18]$, attesting to the universal nature of heterogeneity associated with the avian and reptilian $\delta$-crystallins. All subunit bands shown in the isoelectric-focusing gels may represent mainly the post-translational modified products of $\delta$-crystallin genes, since there are only two gene loci in the chicken and duck $[13,24,25]$ to account for multiple isomeric forms of this crystallin. Recently, we have demonstrated that chicken and duck $\delta$-crystallins possess inherent heterogeneities associated with their native forms and subunits [7]. Our data also indicated that all multiple native isoforms of duck $\delta$-crystallin possessed genuine argininosuccinate-lyase activity, as evidenced by the activity staining in native isoenzyme electrophoresis. The possibilities that these multiple components may be due to posttranslational modifications, such as deamidation, glycosylation and phosphorylation are currently under investigation.

\section{Identification of glycogen by carbohydrate analysis}

The presence of glycogen in lenses has been studied by histochemical methods by which quantitative estimation is difficult. Raman spectroscopy is well-suited for the in situ estimation of this polymeric carbohydrate because the Raman signals due to glycogen are strong and can be readily measured [26]. The best indicator for the presence of glycogen is the Raman line at $481 \mathrm{~cm}^{-1}$, where protein vibrations do not normally interfere. We have corroborated the identifjcation of this glycogen peak by near-IR Fourier transform Raman spectroscopy (Refs. 27,28 and unpublished results). The pioneering study of lens glycogen by Rabaey [21] showed that glycogen constitutes about $3 \%$ of the wet weight and more than $10 \%$ of the dry substance (about 19\%) of the pigeon lens. In our analysis of sugar content by phenol-sulfuric acid method [11], using sucrose as standard about $14 \%$ of the total dry lens extract was found to be polymeric glycogen. In contrast, the high-molecular-mass peak from gel filtration column (peak 1 of Fig. 1) was found to contain more than $95 \%$ sugar. Therefore, the low UV-absorbing material in this peak is clearly identified as glycogen by sugar analysis when coupled with the Raman spectroscopic data. Glycogen, a polysaccharide used as a reserve source of energy in the cell, is abundant in certain types of tissues such as liver and muscle. The biological function and significance for the presence of a high content of glycogen in the pigeon lens deserve further study in the future.

A summary of physicochemical properties of the crystallin components and glycogen of pigeon lenses is given in Table I for reference and comparison with other lens systems.

\section{Amino-acid and sequence analysis of pigeon crystallins}

In order to have a more defined and meaningful comparison of various crystallins, we have carried out the purification of each crystallin peak of Fig. 1 on anion-exchange chromatography (data not shown) essentially according to the same procedure used for the isolation and subsequent separation of $\delta$-crystallin from the contamination of $\beta$-crystallins $[5,6]$. The amino-acid compositions of various purified crystallins are shown in Table II. It is noteworthy that the molecular sizes, subunit compositions coupled with the amino-acid compositions of each crystallin class in the pigeon lens 
TABLE I

\section{Physicochemical properties of pigeon lens components}

The methods used for determination of native and subunit molecular masses are described in Materials and Methods. Estimation of percentage yield was based on the absorption-peak areas of four fractions (Pg-1-4) in Fig. 1 without taking into account of the different molar extinction coefficients for glycogen and different crystallins. Chemical analysis was based on sugar and amino-acid analysis on the lyophilized samples of these four fractions. Enzymic activity refers to the assays performed for argininosuccinate lyase activity and that of Pg-3 was found to be below $0.001 \mathrm{unit} / \mathrm{mg}$ as compared to 0.23 unit $/ \mathrm{mg}$ of duck $\delta$-crystallin.

\begin{tabular}{|c|c|c|c|c|}
\hline Property & $\mathrm{Pg}-1$ & $\mathrm{Pg}-2$ & $\mathrm{Pg}-3$ & $\mathrm{Pg}-4$ \\
\hline Yield & 6.4 & 34.6 & 20.3 & 38.7 \\
\hline Native & $>200$ & 450 & 200 & 110 \\
\hline Subun & & 20,22 & $50,23-33$ & $323-28$ \\
\hline Chemical a & $>950$ & r protein & protein & protein \\
\hline Enzyme activity & none & none & very low & none \\
\hline
\end{tabular}

are found in general to be similar to those orthologous ones of other well-characterized mammalian species [1-3].

$\mathrm{N}$-terminal sequence analyses of these purified crystallin fractions isolated from TSK DE-650 anion-exchange column by Edman degradation were carried out in the microsequencing sequenator. They were all found to yield no $\mathrm{N}$-terminal residues upon repeated

\section{TABLE II}

\section{Comparison of amino-acid compositions of crystallins}

Data of amino-acid compositions are means of duplicate analyses and expressed as mol\%. The abbreviations $\mathrm{Os}$ and $\mathrm{Pg}$ represent ostrich and pigeon, respectively, with the Greek symbols indicating three major crystallin classes, i.e., $\alpha$-, $\beta$ - and $\delta$-crystallins. Data for ostrich and chicken crystallins are taken from Refs. 6 and 29. The hydrolysis condition for the determination of Trp is microwave heating using $4 \mathrm{M}$ methanesulfonic acid containing $0.2 \%$ 3-(2aminoethyl)indole [10].

\begin{tabular}{lrrrrrrr}
\hline Amino acid & Os- $\alpha$ & Pg- $\alpha$ & Os- $\beta$ & Pg- $\beta$ & Os- $\delta$ & Pg- $\delta$ & Chick- $\delta$ \\
\hline 1/2 Cys & 0.8 & 1.0 & 1.0 & 1.2 & 0.3 & 0.2 & 0.0 \\
Asx & 9.1 & 8.9 & 8.8 & 8.5 & 8.2 & 7.8 & 7.1 \\
Thr & 3.6 & 3.1 & 3.1 & 2.7 & 7.1 & 6.0 & 7.6 \\
Ser & 10.1 & 10.5 & 7.1 & 6.8 & 9.2 & 9.5 & 10.1 \\
Glx & 10.7 & 10.3 & 16.4 & 15.1 & 11.7 & 10.5 & 11.4 \\
Pro & 7.0 & 6.7 & 5.3 & 5.5 & 3.1 & 4.1 & 2.5 \\
Gly & 6.3 & 6.8 & 9.1 & 9.8 & 5.9 & 5.2 & 5.6 \\
Ala & 4.6 & 5.1 & 5.5 & 5.7 & 9.1 & 8.6 & 8.3 \\
Val & 4.9 & 5.3 & 5.9 & 5.5 & 6.7 & 6.0 & 6.9 \\
Met & 2.0 & 2.2 & 1.7 & 2.4 & 2.0 & 2.4 & 2.2 \\
Ile & 6.4 & 6.1 & 4.6 & 5.3 & 7.4 & 7.8 & 8.1 \\
Leu & 8.5 & 7.7 & 5.9 & 5.4 & 12.9 & 13.5 & 14.5 \\
Tyr & 2.5 & 2.3 & 3.1 & 3.7 & 0.3 & 1.4 & 1.1 \\
Phe & 6.2 & 7.0 & 4.5 & 3.1 & 3.3 & 3.4 & 2.2 \\
His & 3.9 & 3.5 & 3.3 & 2.8 & 1.4 & 2.2 & 1.3 \\
Lys & 5.6 & 6.1 & 5.8 & 6.5 & 6.6 & 6.2 & 6.5 \\
Arg & 7.4 & 6.6 & 6.1 & 6.9 & 4.7 & 4.9 & 4.3 \\
Trp & 0.5 & 0.7 & 3.2 & 3.0 & 0.3 & 0.5 & 0.2 \\
\hline
\end{tabular}

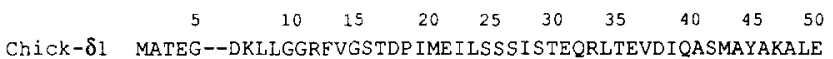

Pigeon $\delta$ MASEG--DKMLGGREVGSTDPVMEMLSASITIDQRLAEVDIQGSMAYAKALE DUCk- $\delta 2$ MASEARGDKLWGGRF SGSTDP IMEKLNSSIAYDQRLSEVDIQGSMAYAKALE

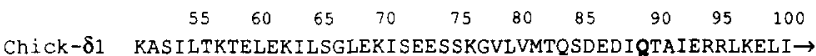
Pigeon $\delta$ KAGILSKSELEKTLSGLEKISEEWSKGVEVVTPTDEDIBTANERRLKELI $\rightarrow$ DuCK $-\delta 2 \quad$ KAGILTKTELEKILSGLEKISEEWSKGVEVVKQSDED IBTANERRLKELI $\rightarrow$

Fig. 4. Comparison of $\mathrm{N}$-terminal sequences of chick, pigeon and duck $\delta$-crystallins. The sequence for pigeon $\delta$-crystallin was obtained by PCR-amplification of the cDNA constructed from mRNA of fresh 1 -month-old pigeon lenses. Those sequences listed for chick and duck crystallins were taken from Refs. 29, 32 and 13, respectively. Note that a His residue was identified as the 89 th amino acid of the pigeon sequence, same as duck $\delta$-crystallin with enzyme activity. However, a pigeon sequence similar to that of chick $\delta$-crystallin lacks the inserted Ala-Arg between residues 4 and 5. Amino-acid residues are denoted by one-letter symbols.

trials, indicative of blocked amino-termini in all these crystallins.

Sequence analysis by PCR and structural comparison of avian $\delta$-crystallins

We have therefore resorted to the analysis of protein sequence by molecular cloning of cDNAs constructed from total mRNA of the fresh pigeon lenses and subsequent PCR amplification of CDNA encoding $\delta$-crystallin with the aim of obtaining important sequence information for structural comparison with those $\delta$-crystallins of other species.

The DNA sequence determined corresponds to the complete coding regions of 466 amino-acid residues, including the initiating methionine residue. The determined nucleotide sequence ${ }^{1}$ showed greater than $85 \%$ similarity to the corresponding parts of those $\delta$-crystallin genes of chicken and duck lenses [13,29], underlying the closely-related phylogenetic relationship between these proteins. On the deduced protein sequence level of the first $\mathrm{N}$-terminal 100 amino-acid sequences (Fig. 4 ), the number of nonidentical amino-acid residues is about 20 and 19 for the pair-wise comparison of chicken/pigeon and duck/pigeon, respectively. In our assays of argininosuccinate lyase activity by monitoring the absorbance change at $240 \mathrm{~nm}$ due to the formation of fumarate $[6,7]$, the lens extract and purified $\delta$-crystallin of pigeon exhibited about similar specific activity to that of chicken lens extract which has been shown to be almost devoid of enzymic activity when compared to those exhibited by duck and goose lenses [8]. It is to be noted that active $\delta$-crystallins isolated from several avian species including duck, goose and ostrich [6-8] all can maintain their activities in spite of their long storage ( $>6$ months) in lyophilized forms (at $4^{\circ} \mathrm{C}$ ). Actually, we have applied the stable duck $\delta$-crystallin 
in the large-scale synthesis of argininosuccinate with success [30]. Therefore, it would be of great interest to compare the primary sequences of the active (duck) and inactive (chicken and pigeon) $\delta$-crystallins in order to locate the active amino-acid residues responsible for the enzymic activity. In the analysis and comparison of $\delta$-crystallins and authentic argininosuccinate lyase, Barbosa et al. [31] have suggested that His-89 $\rightarrow$ Gln substitution in chicken $\delta$-crystallin as compared to that of active duck crystallin might account for the loss of enzymic activity in the former species. As shown in Fig. 4 , the inactive pigeon $\delta$-crystallin possesses a His residue at this position similar to that in active duck protein, this may suggest that His- 89 is not the only critical residue essential for the enzymic activity of $\delta$-crystallin *. In contrast to the conclusion of Wistow and Piatigorsky [13], who suggested that the inserted sequence of Ala-Arg in the duck $\delta_{2}$-crystallin between positions 4 and 5 of the chick and pigeon sequences is not of critical importance for enzyme function, we believe that this inserted ARGD segment may be the major structural difference between active duck and inactive pigeon $\delta$-crystallins. Site-specific mutagenesis for the construction of this mutant with the inserted sequence on the pigeon cDNA clone is currently in progress to assess the role of this mutation on the enzyme activity.

Screening and immunological comparison of $\delta$-crystallins from different species

Since there are great differences regarding the enzymic activity of $\delta$-crystallins isolated from different species, we have analyzed the antigenic structures of these structurally similar crystallins using the antiserum against purified duck $\delta$-crystallin isolated from the subunit protein bands in SDS-PAGE. For immunoblotting detection of $\delta$-crystallins with this antibody (Fig. 5), the gels were subjected to electroblotting on a nitrocellulose membrane after SDS-PAGE. It is noteworthy that antiserum against duck $\delta$-crystallin did cross-react specifically only with $\delta$-crystallins of different sources and no reactions were detected with other crystallin classes, such as $\alpha$ - and $\beta$-crystallins. It also reacted strongly with the authentic bovine liver argininosuccinate lyase, corroborating the high sequence homology between these two proteins. These observations are consistent with the existence of common antibodybinding domains in these related crystallins. However, in our previous immunodiffusion according to Ouchterlony's method [8], a complete cross-reaction between $\delta$-crystallins from duck and goose lenses is found, in contrast to the much weaker reaction observed be-

\footnotetext{
* The complete nucleotide and protein sequences of pigeon $\delta$-crystallin will be published in FEBS Letters (Lin, C.-W. and Chiou, S.-H. (1992) FEBS Lett., in press).
}

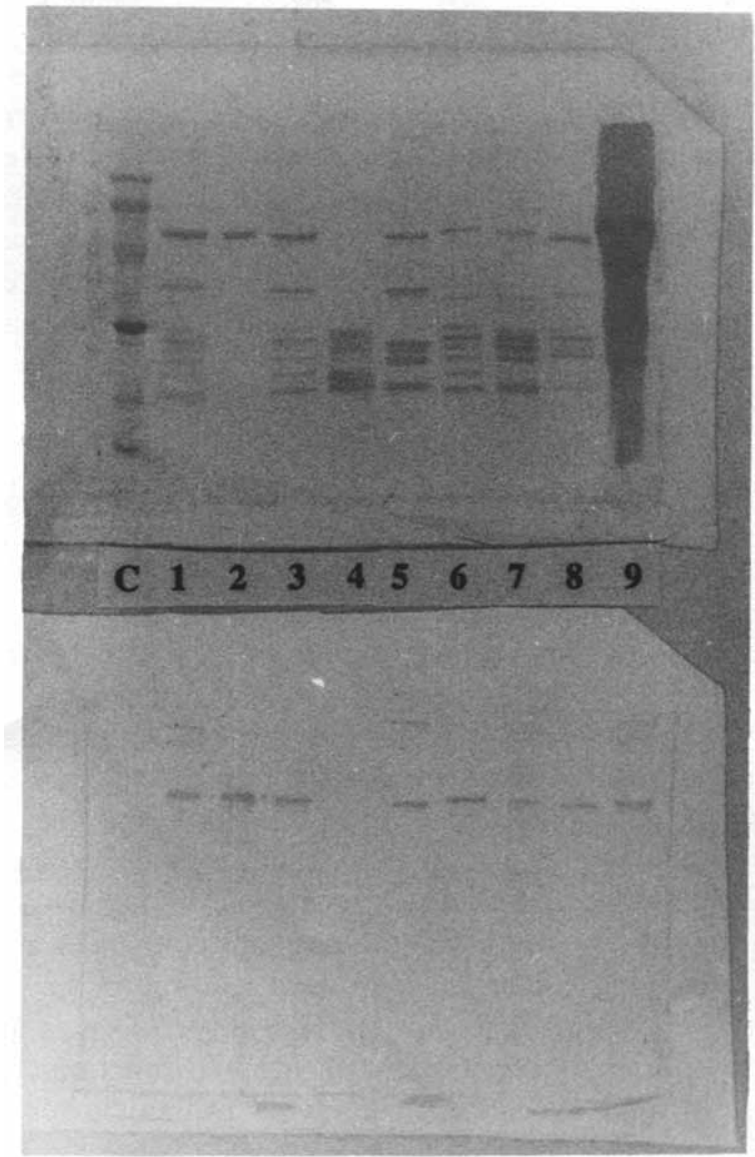

Fig. 5. Immunoblotting analysis of the antiserum against duck $\delta$ crystallin to various lens extracts. The gels were subjected to electroblotting to a nitrocellulose membrane after SDS-PAGE followed by immunological analysis using rabbit antiserum against duck $\delta$ crystallin and stained with horseradish peroxidase conjugated goat anti-rabbit IgG. Lane C, standard protein markers in $\mathrm{kDa}$ : phosphorylase $b(94)$, bovine serum albumin (66), ovalbumin (45), carbonic anhydrase (30), soybean trypsin inhibitor (20) and lysozyme (14). Lanes shown: 1 , duck lens extract; 2 , duck $\delta ; 3$, goose lens extract; 4 , shark lens extract; 5 , caiman lens extract; 6 , pigeon lens extract; 7 , ostrich lens extract; 8 , chicken lens extract and 9, argininosuccinate lyase of bovine liver. Top, Coomassie-blue-stained gel; bottom, immunoblot of the SDS-PAGE gel with antiserum against purified duck $\delta$-crystallin.

tween duck and chicken $\delta$-crystallin. This would indicate that duck and chicken $\delta$-crystallins are structurally related, yet the surface epitopes for recognizing the antibody are somewhat different between active enzymic $\delta$-crystallins (duck and goose) and inactive one (chicken). We have also compared immunological cross-reactions of various lens extracts from the representative species from Reptilia (caiman), Pisces (shark), Amphibia (bullfrog) and Mammalia (pig). The result corroborated previous observations $[18,20,22]$ that only reptiles and birds contained $\delta$-crystallins. The evolutionary significance for the existence of abundant enzymic $\delta$-crystallins in certain species of Aves and Reptilia in the animal kingdom poses an interesting topic for future molecular evolution study. 


\section{Conclusions}

A systematic and general approach has been carried out to provide some basic information on the lens composition of pigeon eye lenses. Some salient differences between pigeon and other avian species have been found, notably regarding high glycogen content and peculiar $\delta$-crystallin variation with aging. We have established the amino-acid sequence of $\delta$-crystallin by construction and sequencing of cDNA by virtue of PCR DNA-amplification technique to circumvent the inherent difficulty of protein sequencing due to the presence of blocked amino-terminal groups in all pigeon crystallins. The sequence comparison between enzymically active duck and inactive chicken and pigeon $\delta$-crystallins seemed to contradict the claim that the His- $89 \rightarrow$ Gln mutation in the chicken $\delta$-crystallin may account for the loss of argininosuccinate lyase activity in this avian species as compared to high enzymic activity in the duck crystallin. Further studies on the expression of pigeon $\delta$-crystallin in some cell culture systems coupled with site-specific mutagenesis of the amplified clones may be conducive to unraveling the intriguing evolutionary process leading to the loss of this crystallin in certain lineages of vertebrates and the predominant recruitment of this dual-function crystallin in some species of avian and reptilian classes.

\section{Acknowledgement}

This work was supported in part by Academia Sinica and the National Science Council (NSC Grant 810203-B001-04), Taipei, Taiwan.

\section{References}

1 Chiou, S.-H. (1986) FEBS Lett. 201, 69-73.

2 De Jong, W.W. and Hendriks, W. (1986) J. Mol. Evol. 24, 121-129.

3 Harding, J.J. and Dilley, K.J. (1976) Exp. Eye Res. 22, 1-73.

4 Wistow, G. and Piatigorsky, J. (1988) Annu. Rev. Biochem. 57, $479-504$.

5 Chiou, S.-H., Lee, H.-J. and Chang, G.-G. (1990) Biochem. J. $267,51-58$.
6 Chiou, S.-H., Lo, C.-H., Chang, C.-Y., Itoh, T., Kaji, H. and Samejima, T. (1991) Biochem. J. 273, 295-300.

7 Lee, H.-J., Chiou, S.-H. and Chang, G.-G. (1992) Biochem. J. $283,597-603$.

8 Chiou, S.-H., Lee, H.-J., Chu, H., Lai, T.-A. and Chang, G.-G. (1991) Biochem. Int. 25, 705-713.

9 Laemmli, U.K. (1970) Nature 227, 680-685.

10 Chiou, S.-H. and Wang, K.-T. (1990) in Current Research in Protein Chemistry (Villafranca, J.J., ed.), pp. 3-10, Academic Press, New York.

11 Dubois, M., Gilles, K.A., Hamilton, J.K., Robers, P.A. and Smith, F. (1956) Anal. Chem. 28, 350-356.

12 Chiou, S.-H., Chylack, L.T., Jr., Tung, W.H. and Bunn, H.F. (1981) J. Biol. Chem. 256, 5176-5180.

13 Wistow, G.J. and Piatigorsky, J. (1990) Gene 96, 263-270.

14 Sanger, F., Milklen, S. and Coulson, A.R. (1977) Proc. Natl. Acad. Sci. USA 74, 5463-5467.

15 Chiou, S.-H. (1984) J. Biochem. 95, 75-82.

16 Chiou, S.-H., Chang, W.-C., Pan, F.-M., Chang, T. and Lo, T.-B. (1987) J. Biochem. 101, 751-759.

17 Chiou, S.-H. (1987) Int. J. Peptide Protein Res. 30, 108-116.

18 Chiou, S.-H., Chang, W.-P. and Lo, C.-H. (1988) Biochim. Biophys. Acta 955, 1-9.

19 Chiou, S.-H., Chang, W.-P., Ting, L.-M., Lai, T.-A. and Lin, H.-K. (1988) Curr. Eye Res. 7, 1017-1022.

20 Clayton, R.M. (1974) in The Eye, Vol. 5 (Davson, H. and Graham, L.T., eds.), pp. 399-494, Academic Press, London.

21 Rabaey, M. (1963) Nature 198, 206-207.

22 Rabaey, M. (1962) Exp. Eye Res. 1, 310-316.

23 Chiou, S.-H., Chang, W.-P. and Lai, T.-A. (1989) Curr. Eye Res. $8,1055-1061$.

24 Nickerson, J.M., Wawrousek, E.F., Borras, T., Hawkins, J.W., Norman, B.L., Filpula, D.R., Nagle, J.W., Ally, A.H. and Piatigorsky, J. (1986) J. Biol. Chem. 261, 552-557.

25 Piatigorsky, J., O'Brien, W.E., Norman, B.L., Kalumuck, K., Wistow, G.J., Borras, T., Nickerson, J.M. and Wawrousek, E.F. (1988) Proc. Natl. Acad. Sci. USA 85, 3479-3483.

26 Yu, N.-T., East, E.J., Chang, R.C.C. and Kuck, J.F.R., Jr. (1977) Exp. Eye Res. 24, 321-334.

27 Chiou, S.-H., Lee, B.-S., Chang, C.-C. and Yu, N.-T. (1991) Biochem. Int. 25, 387-395.

28 Chiou, S.-H., Lee, B.-S. and Yu, N.-T. (1992) Biochem. Int. 26, $747-758$

29 Nickerson, J.M. and Piatigorsky, J. (1984) Proc. Natl. Acad. Sci. USA 81, 2611-2615.

30 Wu, C.-Y., Chen, S.-T., Chiou, S.-H. and Wang, K.-T. (1991) Biotech. Lett. 13, 405-410.

31 Barbosa, P., Cialkowski, M. and O'Brien, W.E. (1991) J. Biol. Chem. 266, 5286-5290.

32 Yasuda, K., Kondoh, H., Okada, T.S., Nakajima, N. and Shimura, Y. (1982) Nucleic Acids Res. 10, 2879-2891. 